\title{
INTRODUCTION
}

\section{Implications of emerging pathogens in the management of haemophilia}

\author{
S. W. PIPE \\ Pediatric Hemophilia and Coagulation Disorders Program, University of Michigan, Women's Hospital, Ann Arbor, MI, USA
}

In the early $1980 \mathrm{~s}$, more than $50 \%$ of persons with haemophilia became infected with human immunodeficiency virus (HIV). This high rate of infectivity was in large part because of the initially limited reaction to the possibility and implications of HIV transmission through blood and blood therapies. Significant morbidity and mortality were the result of this tragically inadequate response. While the risks of HIV transmission are now well documented, clinicians must be aware of the potential for new pathogens.

This supplement is a synthesis of the proceedings of an interdisciplinary symposium convened in August 2005 at Sydney, Australia. The symposium, part of the XXth Congress of the International Society on Thrombosis and Haemostasis, was designed to provide a unique perspective on the state of emerging pathogens and to review proactive measures to prevent their transmission in patients with haemophilia.

New, reemerging or drug-resistant pathogens continue to arise worldwide, and their presence presents an ongoing threat to the blood supply. Recent years have produced outbreaks of severe acute respiratory syndrome (SARS), West Nile virus (WNV) and avian influenzas. In his article, Dr Michael Tapper puts forth the basic definition of an emerging pathogen agreed upon by the medical and scientific communities. Using the examples of WNV, SARS and avian flu, he outlines the factors that contribute to the emergence of these pathogens, including microbial adaptation, global travel and human demographics.

Non-viral disease-causing agents, such as the prions that cause variant Creutzfeldt-Jacob disease

Correspondence: Steven W. Pipe, MD, Pediatric Hemophilia and Coagulation Disorders Program, University of Michigan, Women's Hospital, 1500 East Medical Center Drive, Room L2110, Ann Arbor, MI 48109.

Tel.: +1 734647 2893; fax: +1 734936 7083;

e-mail: ummdswp@med.umich.edu
(vCJD), also pose a real and significant threat to blood and blood-derived therapies, as exemplified by the recent experience in the UK. Dr James Ironside's article reviews the clinical and management characteristics of $\mathrm{vCJD}$ with an emphasis on the risks of disease transmission. He describes the three classes of prion diseases: idiopathic (also known as sporadic), inherited and acquired (the classification for variant CJD). In addition, Dr Ironside reviews the epidemiological considerations, including the theoretical role of genetics in vCJD susceptibility, the potential risks of transmission through blood and blood products and the trends in and extent of its spread to date.

In his article, Dr Gerry Dolan shares the clinician's perspective on the indirect clinical and psychological impact that vCJD may potentially have on patients with haemophilia in the UK, primarily as a result of new recommendations and policies to safeguard patients from transfusion-transmitted infections. Often developed in concert with governmental and non-governmental agencies, these policies describe how to inform and manage patients who may have been exposed to vCJD through the use of UK-based plasma concentrates, and therefore may pose a risk for human-to-human transmission. Unfortunately, these policies could have unintended consequences for the haemophilia population: potential stigmatisation and interference with necessary medical and surgical care. The emergence of vCJD argues for the utmost caution in the treatment of persons with haemophilia, both medically and sociopolitically.

By their very nature, unknown emerging pathogens will always cause uncertainty within the medical and scientific communities, but as evidenced by the HIV crisis of the 1980s, hesitation in the face of theoretical risks can result in increased morbidity and mortality. My article describes the importance of both anticipating the potential risks which emerging pathogens may pose to patients with haemophilia, and discussing these risks, as well as the relative 
merits of available therapies with patients. Practitioners must understand the real and theoretical risks to their patients with haemophilia and be able to address those fears and anxieties appropriately. I review the basic information to know when discussing emerging pathogens with a haemophilia patient. While it is likely that emerging pathogens will always be with us, they represent an opportunity for an open and frank discussion between practitioner and patient regarding optimal therapy for this disease and other bleeding disorders.

A synthesis of a question and answer session about emerging pathogens, patient management, vCJD and the impact of vCJD within the haemophilia community follows these four articles. The contents of this supplement contain the most current information on identified and potential infectious disease risks, their mechanisms for emergence, and their associated threat to the safety of the blood supply worldwide. A special emphasis is placed on the impact of emerging infectious diseases on clinical practice in haemophilia. A thorough grasp of this information enables clinicians to proactively review therapeutic regimens with their haemophilia patients in order to minimize anxiety with regard to emerging infectious disease, counsel them on the current safety of plasma-derived therapies and recommend a pharmacological course of therapy that will result in the most optimal outcomes possible. 